\title{
O ORDENAMENTO DE UMA NOVA PESCARIA DIRECIONADA AO PEIXE-SAPO (Lophius gastrophysus) NO SUDESTE E SUL DO BRASIL
}

\author{
PEREZ, J.A.A.; PEZZUTO, P.R.; ANDRADE, H.A.; SCHWINGEL, P.R.; RODRIGUES-RIBEI- \\ RO, M. \& R. WAHRLICH \\ Centro de Educação Superior de Ciências Tecnológicas da Terra e do Mar - CTTMar/ \\ UNIVALI, C.P. 360, CEP 88302-202, Itajaí, SC \\ e-mail: angel@cttmar.univali.br
}

\begin{abstract}
RESUMO
A pescaria do peixe-sapo (Lophius gastrophysus) desenvolveu-se no Sudeste e Sul do Brasil através da atividade multiespecífica da frota de arrasteiros operando a mais de $100 \mathrm{~m}$ de profundidade e de uma pescaria de emalhe dirigida, composta por embarcações estrangeiras arrendadas especializadas na captura e processamento a bordo desse pescado. Devido ao crescente e descontrolado interesse por essa espécie durante 2001, o Departamento de Pesca e Aqüicultura (Ministério da Agricultura, Pecuária e Abastecimento, MAPA) desencadeou um processo de ordenamento dessa pescaria, abrangendo as fases de (a) coleta de informações tecnológicas, biológicas e pesqueiras, (b) avaliação do estoque e da pescaria, (c) apresentação para o setor produtivo, governamental e científico, da referida avaliação e das subseqüentes recomendações para o ordenamento e, finalmente, (d) tomada decisão sobre medidas de ordenamento da pescaria a serem implementadas em 2002, dentro do Comitê Permanente de Gestão (CPG) de Recursos Demersais de Profundidade. O presente trabalho apresenta um resumo desse processo, executado pelo Grupo de Estudos Pesqueiros do CTTMar-UNIVALI dentro do âmbito do convênio UNIVALI/MAPA, incluindo um diagnóstico da pescaria do peixe-sapo em 2001, uma descrição dos procedimentos e critérios adotados para a estimativa de níveis sustentáveis de explotação do recurso e, finalmente, um rol de recomendações objetivas direcionadas ao ordenamento da pescaria para o ano de 2002. Por último descreve-se o evento de apresentação pública dos dados levantados e das recomendações como parte culminante de um inédito processo dinâmico e participativo de ordenamento pesqueiro no Brasil
\end{abstract}

Palavras-chave: Peixe-sapo, Lophius gastrophysus, Recurso Demersal de Profundidade, Manejo pesqueiro, Avaliação de Estoque, Sudeste e Sul do Brasil.

\section{MANAGEMENT OF A NEW MONKFISH (Lophius gastrophysus) FISHERY IN SOUTHERN BRAZIL}

\begin{abstract}
A monkfish (Lophius gastrophysus) fishery developed in southern Brazil during 2001 through the multispecific deep-water operations ( $>100 \mathrm{~m}$ depth) of a national trawling fleet and through the gill net fishing operations conducted by a specialized chartered foreign fleet. Due to the increasing interest in such resource, the Department of Fishing and Aquaculture (Ministry of Agriculture) launched a management initiative that included (a) the collection of biological and technological information, (b) the assessment of the stock and the fishery, (c) the public debate of these assessments with the Brazilian fishing industry, government and scientists and, finally, (d) the definition of management actions for the 2002 fishing season within a Permanent Committee established for the management of deep-water demersal resources (Ministry of Agriculture). The present work summarizes this management initiative mostly conducted by CTTMar-UNIVALI fishery
\end{abstract}




\begin{abstract}
science group under a scientific cooperation program with the Ministry of Agriculture. It includes a diagnosis of the monkfish fishery in 2001, a description of procedures and criteria adopted for the estimate of the resource sustainable levels of exploitation and, finally, a list of objective recommendations as subsidies to the definition of the management actions for the 2002 fishing season. Lastly a public presentation and debate of the information raised and the proposed recommendations is described as the culmination of a dynamic and participative unseen process of fishing management in Brazil.
\end{abstract}

Key words: Monkfish, Lophius gastrophysus, deep-water demersal resource, fishing management, stock assessment, southern Brazil.

\section{INTRODUÇÃO}

A expansão da pesca industrial no Sudeste e Sul do Brasil para áreas de talude e águas profundas tomou impulso em 2001 a partir de dois processos paralelos de desenvolvimento pesqueiro. O primeiro foi um processo natural de deslocamento da frota nacional de arrasteiros para áreas em profundidades superiores a $100 \mathrm{~m}$, observado principalmente nos estados do Rio de Janeiro e Santa Catarina (Perez et al., 2001a). O segundo foi decorrência da implantação do plano de ocupação da ZEE brasileira, lançado pelo Departamento de Pesca e Aqüicultura (DPA) do Ministério da Agricultura, Pecuária e Abastecimento (MAPA), cujo principal instrumento foi o incentivo ao processo de arrendamento de embarcações estrangeiras por empresas nacionais.

Regulamentado pelo Decreto Federal num. 2840/98 de 10 de novembro de 1998, o arrendamento viabilizou a operação de embarcações estrangeiras em áreas pouco conhecidas e sobre espécies pouco explotadas ou inexplotadas até então pela frota nacional, tendo como objetivos principais (a) a avaliação da rentabilidade e do potencial de sustentabilidade da pesca profunda nas regiões Sudeste e Sul e (b) a introdução de novas tecnologias de captura e processamento de pescado. Para tanto, as embarcações arrendadas foram submetidas a exigências que garantiram a geração de informações detalhadas de todas as operações de pesca, com destaque para a manutenção obrigatória de sistemas de rastreamento viasatélite e de observadores de bordo (Rodrigues-
Ribeiro, 2001; Wahrlich, 2001). Gerenciadas por pesquisadores do Centro de Ciências Tecnológicas da Terra e do Mar (CTTMar) da Universidade do Vale do Itajaí (UNIVALI) em convênio com o DPA-MAPA (MAPA/SARC/DPA 03/2001) as informações provenientes da atividade pesqueira em águas profundas, tanto da frota nacional quanto daquela arrendada, têm servido com base para o desenvolvimento de estudos científicos que, por sua vez, têm gerado subsídios para o desenvolvimento sustentável da pesca profunda no Brasil (Perez et al., 2001b).

O peixe-sapo (Lophius gastrophysus) vem se destacando desde meados de 2000 , como um dos principais alvos da pesca de profundidade no Sudeste e Sul do Brasil (Perez et al., 2001a), motivando, pelo seu elevado valor no mercado internacional, o direcionamento de uma importante parcela da frota de arrasteiros nacionais, tradicionalmente atuantes sobre a plataforma continental, e o desenvolvimento de uma pescaria de emalhe dirigida, composta por embarcações estrangeiras arrendadas especializadas na captura e processamento a bordo desse pescado (Perez et al., 2001b). A partir do crescente e descontrolado interesse por essa espécie durante 2001, intensificaramse da mesma forma, por parte do setor produtivo e governamental, as expectativas de projeção e sustentabilidade futura dessa valiosa pescaria.

Assim, por iniciativa do DPA-MAPA, desencadeou-se um processo de ordenamento da pescaria do peixe-sapo, com cerca de 14 meses de execução, abrangendo (a) coleta de 
informações tecnológicas, biológicas e pesqueiras entre janeiro e dezembro de 2001, (b) avaliação do estoque e da pescaria entre janeiro e abril de 2002, (c) uma apresentação para o setor produtivo, governamental e científico, da referida avaliação e das subseqüentes recomendações para o ordenamento e, finalmente, (d) a tomada de decisão sobre medidas de ordenamento da pescaria a serem implementadas em 2002, dentro do Comitê Permanente de Gestão (CPG) de Recursos Demersais de Profundidade estabelecido através da Instrução Normativa num. 2, de 30 de janeiro de 2002, sob a coordenação do MAPA.

As três primeiras etapas desse processo foram executadas pelo Grupo de Estudos Pesqueiros do CTTMar-UNIVALI dentro do âmbito do referido convênio UNIVALI/MAPA e culminaram com a elaboração de relatório final apresentado ao MAPA como subsídio para a última etapa prevista (Perez et al., 2002a). O presente trabalho apresenta uma síntese desse processo, incluindo um diagnóstico da pescaria do peixe-sapo em 2001, uma descrição dos procedimentos e critérios adotados para a estimativa de níveis sustentáveis de explotação do recurso e, finalmente, um rol de recomendações objetivas direcionadas ao ordenamento da pescaria para o ano de 2002. Por último descreve-se o evento de apresentação pública dos dados levantados e das recomendações como parte culminante de um inédito processo dinâmico e participativo de ordenamento pesqueiro no Brasil.

\section{DIAGNÓSTICO DO ESTADO DA PESCARIA}

A pescaria do peixe-sapo Lophius gastrophysus, no Sudeste e Sul do Brasil atingiu um impulso sem precedentes durante 2001. Alguns anos antes apenas registros de desembarques comerciais realizados pela frota de arrasteiros sediada principalmente no Rio de Janeiro e Santa Catarina, demonstravam um interesse crescente pela espécie como respos- ta a oportunidades de negócios no mercado internacional (Perez et al., 2001b).

Durante 2001 construiu-se um esforço de pesca sobre a espécie a partir principalmente de operações multiespecíficas de uma numerosa frota nacional de arrasteiros duplos e de nove embarcações estrangeiras de pesca de emalhe arrendadas por empresas nacionais (Wahrlich \& Rodrigues-Ribeiro, 2002). Este esforço pôde ser estimado em mais de 100.000 horas de arrasto e pelo lançamento de 611.028 redes de emalhe que somaram cerca de 179.870 horas de imersão, sendo distribuído entre as isóbatas de 100 a 740 m, desde Cabo Frio (RJ) até o limite sul da ZEE brasileira (latitudes $21^{\circ} \mathrm{S}-34^{\circ} \mathrm{S}$ ), particularmente em cinco áreas de pesca situadas em concavidades topográficas da margem da plataforma continental (Perez et al., 2002b).

Em 2001, os desembarques de peixesapo na região foram estimados em 8.600 toneladas, o equivalente a pouco mais de US\$ $20.700 .000,00$. Dessa produção participaram mais de 200 arrasteiros nacionais (principalmente sediados no Rio de Janeiro e em Itajaí) que contribuíram com cerca de $60 \%$ do desembarque em peso e $70 \%$ do rendimento econômico total, e os desembarques da frota arrendada de emalhe que, com apenas nove embarcações, contribuiu com $37 \%$ dos desembarques e $30 \%$ do rendimento econômico total (Perez et al., 2002a). Estas últimas embarcações foram as que individualmente obtiveram os maiores benefícios econômicos, sobretudo por sua capacidade de estocagem e agregação de valor ao pescado, que foi processado e congelado a bordo atingindo qualidade superior ao produzido pela frota nacional (Wahrlich \& Rodrigues-Ribeiro, 2002) e, conseqüentemente, sendo vendido a preços quase três vezes maiores no mercado internacional, principalmente europeu.

Através da aplicação de modelos populacionais que levaram em conta a composição de tamanhos dos peixes capturados, parâmetros de crescimento, seletividade das 
redes e primeira maturação, preliminarmente estimados para L. gastrophysus ou encontrados na literatura para outras espécies congenéricas do Atlântico (principalmente $L$. budegassa do Atlântico NE), estimou-se uma biomassa média total em 2001 em torno de 61.000 t (Schwingel \& Andrade, 2002; Perez et al., 2002c). Apesar das incertezas envolvidas nessa estimativa, infere-se que a captura total no ano de 2001, incluindo o possível rejeito, tenham resultado de uma taxa de remoção de cerca de $16 \%$ do estoque total. Por outro lado, considerando que a atividade, principalmente da frota de emalhe, concentrou-se em áreas restritas e de elevada concentração do recurso, dentro dessas áreas a capacidade instalada gerou remoções significativamente maiores em 2001, chegando a $60 \%$ da biomassa original (Perez et al., 2002c). Essas depleções localizadas foram consistentemente detectadas nos dados analisados e condicionaram a dinâmica das embarcações de pesca de emalhe, as quais apenas mantiveram patamares satisfatórios de rendimento a partir da alternância das áreas de pesca visitadas durante o ano. Para a maior parte dessa frota, a rotatividade de áreas foi induzida por uma determinação das portarias de autorização de arrendamento, trazendo benefícios tanto para o desempenho individual quanto para a coleta de informações populacionais e pesqueiras (Perez et al., 2002b).

A capacidade estimada de remoção do estoque de peixe-sapo no Sudeste e Sul durante 2001 teve ainda particularidades populacionais relevantes. A pesca de arrasto duplo concentrou seu poder de captura sobre indivíduos menores que $50 \mathrm{~cm}$ de comprimento total, os quais incluíram principalmente machos juvenis e adultos, e fêmeas juvenis. Por outro lado, a pesca de emalhe capturou quase que exclusivamente fêmeas maiores que $50 \mathrm{~cm}$ e sexualmente maturas (Schwingel \& Andrade, 2002). Estes padrões diferenciados são o fruto das interações entre a seletividade dos diferentes métodos de pesca e as características biológicas do estoque, incluindo a estratificação batimétrica dos indivíduos de diferentes tamanhos (menores e imaturos nas áreas mais rasas - maiores e maturos nas áreas profundas) e os diferentes ritmos de crescimento entre machos e fêmeas. Infere-se assim que devido à significativa capacidade de remoção da população por parte dos arrasteiros nacionais, uma grande parcela da captura total do recurso em 2001, tenha sido composta por indivíduos que não participaram de nenhum evento reprodutivo (Schwingel \& Andrade, 2002). Soluções tecnológicas demonstradas principalmente pelos arrasteiros estrangeiros arrendados, que contribuíram com capturas relativamente modestas de peixe-sapo apesar de atuarem nas mesmas áreas, apontaram direções no sentido de minimizar a captura de juvenis pela pesca de arrasto de uma forma geral (Wahrlich \& Rodrigues-Ribeiro, 2002).

A pesca de emalhe foi direcionada ao peixe-sapo, o qual representou o principal componente capturado e processado. No entanto essa atividade ocasionou a captura de fauna acompanhante que, principalmente devido ao elevado esforço resultante das operações com redes de emalhe, pode ter exercido um impacto relevante sobre algumas populações em particular, bem como ao ecossistema profundo como um todo. Destaca-se a mortalidade estimada de 431.786 caranguejos-de-profundidade (Chaceon notialis e C. ramosae) e 8.300 chernes-poveiros (Polyprion americanus) que, ao lado da merluza (Merlucius hubbsi), galode-profundidade (Zenopsis conchifer) e abróteade-profundidade (Urophycis cirrata), são explotadas por outras pescarias direcionadas e multiespecíficas no Sudeste e Sul do Brasil (Perez et al., 2001a; Perez et al., 2001b; Perez et al., 2002b). O caranguejo-de-profundidade, alvo de uma pescaria de covos realizada por duas embarcações arrendadas, tem sido eficientemente capturado pela pesca de emalhe e existem tendências claras de operações direcionadas a essa espécie, principalmente com redes "iscadas", a exemplo do que é realizado em outras áreas de pesca do mundo (Perez et al., 2001b; Bruno et al., 2001). 
O cherne-poveiro é uma espécie plenamente explotada por uma pescaria doméstica de espinhel de fundo cujo aumento do esforço, através do bycatch de outras pescarias profundas, tem sido controlado (Peres \& Haimovici, 1998; DPA/MAPA, 2000). As estimativas de captura incidental de cherne-poveiro pela frota arrendada de emalhe, apontam para uma mortalidade incidental da espécie preocupante frente aos baixos rendimentos da pescaria doméstica de espinhel de fundo, além de caracterizar, devido ao baixo aproveitamento da espécie pelas embarcações de emalhe, um rejeito substancial de uma espécie valiosa (high grading) e com tendência a sobrexplotação (Perez et al., 2002a).

A fauna acompanhante e o rejeito da pesca de arrasto, principalmente dos arrasteiros duplos nacionais, atuantes nas áreas de concentração do peixe-sapo são atualmente desconhecidos. Pode-se generalizar, apenas, neste momento, que provavelmente existe um substancial rejeito de peixe-sapo entre 20 e $40 \mathrm{~cm}$ (a partir de dados coletados por observadores de bordo em arrasteiros arrendados) e diversas espécies de organismos bentônicos e peixes demersais. Esse possível rejeito, no entanto, não deve se sobrepor àquele quantificado para a frota de emalhe, basicamente constituído de organismos maiores.

A atuação das duas frotas participantes na explotação do peixe-sapo, além de outras como a do espinhel de fundo, nas áreas de talude do Sudeste e Sul do Brasil, têm gerado importantes conflitos os quais tendem a se agravar no futuro, principalmente num previsto cenário de redução das taxas de captura de peixe-sapo e potencial aumento da importância de outras espécies para a manutenção de rendimentos satisfatórios nas áreas profundas.

Finalmente, foram detectadas nas operações de pesca de emalhe, práticas de rejeito de resíduos sólidos não-biodegradáveis, com particular destaque para fragmentos de redes de pesca danificadas (Wahrlich \& Rodrigues-
Ribeiro, 2002). Esses fragmentos e principalmente as redes perdidas em função de conflitos com arrasteiros podem prejudicar as áreas de pesca ao continuar capturando peixes e outros organismos (pesca fantasma - ghost fishing). O mesmo pode ser dito em relação às redes deixadas na água por longos períodos, matando uma quantidade desconhecida de organismos que são predados enquanto ficam presos às redes.

\section{PERSPECTIVAS DE SUSTENTABILIDADE DA PESCARIA}

\section{Objetivos de manejo}

A elaboração das bases para medidas de ordenamento da pescaria do peixe-sapo no Sudeste e Sul do Brasil para o ano de 2002 foi fundamentada no diagnóstico do estado da pesca sumarizado acima e foi norteada pelos objetivos básicos de utilização sustentável dos recursos pesqueiros defendidos pelo Estado Brasileiro, bem como por organizações e acordos internacionais dos quais o Brasil é signatário ou membro permanente. Como exemplo citam-se trechos da Constituição Brasileira de 1988 em seu Capítulo VI "Do meio ambiente":

Art. 225. Todos têm direito ao meio ambiente ecologicamente equilibrado, bem de uso comum do povo e essencial à sadia qualidade de vida, impondo-se ao Poder Público e à coletividade o dever de defende-lo e preserva-lo para as presentes e futuras gerações.

$\S 1^{\circ}$ - Para assegurar a efetividade desse direito, incumbe ao Poder Público ... preservare restaurar os processos ecológicos essenciais e prover o manejo ecológico das espécies e ecossistemas;

e também dos itens incluídos no artigo 6 - Princípios Gerais do Código de Conduta para a Pesca Responsável (FAO, 1995): 
6.1. Os estados e os usuários dos recursos aquáticos vivos deveriam conservar os ecossistemas aquáticos. O direito de pescar leva consigo a obrigação de fazê-lo de forma responsável a fim de assegurar a conservação e a gestão efetiva dos recursos aquáticos vivos.

\subsection{A ordenação da pesca deveria fomentar a} manutenção da qualidade, da diversidade e disponibilidade dos recursos pesqueiros em quantidade suficiente para as gerações presentes e futuras, no contexto de segurança alimentar, o alívio da pobreza e o desenvolvimento sustentável. As medidas de ordenamento deveriam assegurar a conservação não apenas de espécies-alvo, mas também aquelas espécies pertencentes ao mesmo ecossistema ou dependentes delas ou que estejam associadas a elas.

6.3. Os Estados deveriam evitar a sobrexplotação e o excesso de capacidade de pesca e deveriam aplicar medidas de ordenamento com o fim de assegurar que o esforço de pesca seja proporcional à capacidade de produção dos recursos pesqueiros e ao aproveitamento sustentável dos mesmos.

6.4. As decisões sobre conservação e ordenamento em matéria de pescarias deveriam estar baseadas nos dados científicos mais fidedignos disponíveis, levando em conta também conhecimentos tradicionais acerca dos recursos e seu hábitat, assim como os fatores ambientais, econômicos e sociais pertinentes.

\subsection{Os Estados e as organizações} subregionais e regionais de ordenamento pesqueiro deveriam aplicar amplamente o critério de precaução na conservação, o ordenamento e a explotação dos recursos aquáticos vivos com o fim de protege-los e de preservar o meioambiente aquático, tomando em consideração os dados científicos mais fidedignos disponí- veis. A falta de informação científica adequada não se deveria utilizar como razão para adiar ou deixar de tomar medidas para conservar as espécies que são objeto da pesca, espécies associadas ou dependentes destas e aquelas que não são objeto da pesca, assim como o meio ambiente.

6.6. ... Os Estados e os usuários dos ecossistemas aquáticos deveriam reduzir ao mínimo o desperdício das capturas tanto das espécies que são objeto da pesca com as que não são ....

Desta forma segue a descrição das medidas que, acredita-se, sejam as mais apropriadas para a sustentação da pescaria a curto e médio prazo, levando-se em conta as informações geradas durante 2001 da pesca nacional e da pesca da frota arrendada, os aspectos teóricos e práticos das experiências de manejo de pescarias demersais, particularmente de espécies do gênero Lophius no Atlântico, e os princípios de precaução (Garcia, 1996).

\section{Cenários de sustentação}

A explotação do estoque de peixe-sapo no Sudeste e Sul do Brasil durante 2001 pode ser abordada sob dois cenários. Inicialmente pode-se assumir um estoque total que continha cerca de $61.000 \mathrm{t}$ das quais, em torno de $16 \%$, entre captura desembarcada e rejeitada, foram removidos durante 2001. Outro cenário, mais realista, considera que a mesma captura total proveio de áreas restritas do talude, onde a atividade removeu efetivamente $60 \%$ da biomassa original. Embora existam possivelmente outras áreas, mais profundas e/ou sobre fundos rochosos ou coralinos, passíveis de ocupação pela frota, parece evidente que as áreas de pesca ocupadas em 2001 merecem uma atenção prioritária no sentido de manterse, dentro delas, uma produção sustentada. Assumir a capacidade de remoção estabelecida sobre um estoque, em sua maior parte, diluído sobre áreas até o momento não identificadas 
como pesqueiros, implicaria em potencializar níveis de exploração que, a priori, promoveriam uma depleção rápida das áreas acessíveis e posteriormente dependeriam da pesca exploratória em áreas pouco conhecidas, pouco densas ou, num cenário ainda pior, sobre espécies rentáveis do bycatch de potencial pesqueiro desconhecido e/ou em conflito com a atividade de outras frotas atuantes na região. Portanto, os níveis de remoção do estoque sugeridos para o período 2002, devem priorizar a atividade no interior das áreas de pesca atualmente explotadas do Sudeste e Sul.

\section{Adoção de Pontos Biológicos de Referência (PBRs)}

Em muitas pescarias emergentes, onde as informações populacionais são insuficientes para a determinação de políticas que otimizem a explotação do recurso, o manejo tem sido praticado através do cálculo de Pontos Biológicos de Referência (PBRs), os quais representam alvos a serem atingidos através da pescaria ou "zonas de perigo" que deveriam ser evitadas pela mesma (Quinn e Deriso, 1999). Nesse sentido, quando alguma informação quantitativa é disponível, esses PBRs podem ser calculados a partir de elementos relativamente simples como, por exemplo, o proposto por Gulland (1971) o qual se baseia na estimativa do Rendimento Máximo Sustentável (RMS) de acordo com a concepção de um modelo de dinâmica de biomassa do tipo Graham-Schaefer (Garcia et al., 1989; Woodby et al., 1993; Caddy, 1999; Quinn \& Deriso, 1999). O PBR especificamente seria o RMS obtido através de uma mortalidade por pesca $\left(F_{m}\right)$ que seria aproximadamente igual à mortalidade natural $M$ atuante no estoque explotado numa situação de equilíbrio. Em termos analíticos o PBR proposto para um determinado ano iseria definido como:

$$
R M S_{t}=F_{m} \cdot B_{t}
$$

e

$R M S=X \cdot M \cdot B_{0}=M \cdot B_{m}$

onde $\mathrm{B}_{0}$ é a biomassa virginal e $X$ é um fator de correção. Assumindo o modelo clássico de crescimento populacional logístico, $X$ seria equivalente a 0,5 (Fig. 1). Além dos pressupostos tradicionais de que a população é denso-dependente e o recrutamento é constante (Woodby et al., 1993; Quinn \& DeRiso, 1999), vários autores têm indicado que $F_{m}$ é de fato menor que $M$ e, portanto, a equação (1) sobreestima RMS quando $X=0,5$ (Fig. 1). Assim, recomenda-se que o fator $X$ deve ser utilizado de uma forma conservativa representando, no máximo, o limite superior de $F_{m}$ (Garcia et al., 1989; Woodby et al., 1993; Quinn \& Deriso, 1999).

A aplicação desse PBR na pescaria do peixe-sapo assumiu que, devido ao fato do estoque estar sendo pouco explotado no Sudeste e Sul antes de 2001, a biomassa estimada da espécie seria próxima $B_{0}$. Também se assumiu $M=0,15$ (variando entre 0,1 e 0,2 ) de acordo com os critérios estabelecidos por Schwingel \& Andrade (2002). O RMS, que corresponderia à captura máxima de peixe-sapo recomendada para o ano de 2002, foi então calculado variando-se o $X$ entre 0 e 0,5 (Fig. 2).

Observa-se que os níveis da captura observados em 2001 só poderiam ser considerados para o ano de 2002 tomando-se como verdadeiro o limite superior do intervalo de confiança $(80 \%)$ da estimativa da biomassa virginal além do valor máximo de $M(M=0,2)$ e o fator $X=0,5$ (Fig. 2). Tomando-se o valor médio de $M(0,15)$ e a mediana da biomassa virginal, no entanto, observa-se que a maior estimativa de RMS em $X=0,5$, seria de cerca de 4.700 t, ou próximo da metade da captura registrada em 2001 (Fig. 2).

Seguindo as recomendações dos autores já mencionados, adotou-se a seguir o valor conservativo de $X=0,27$ o que determina uma 


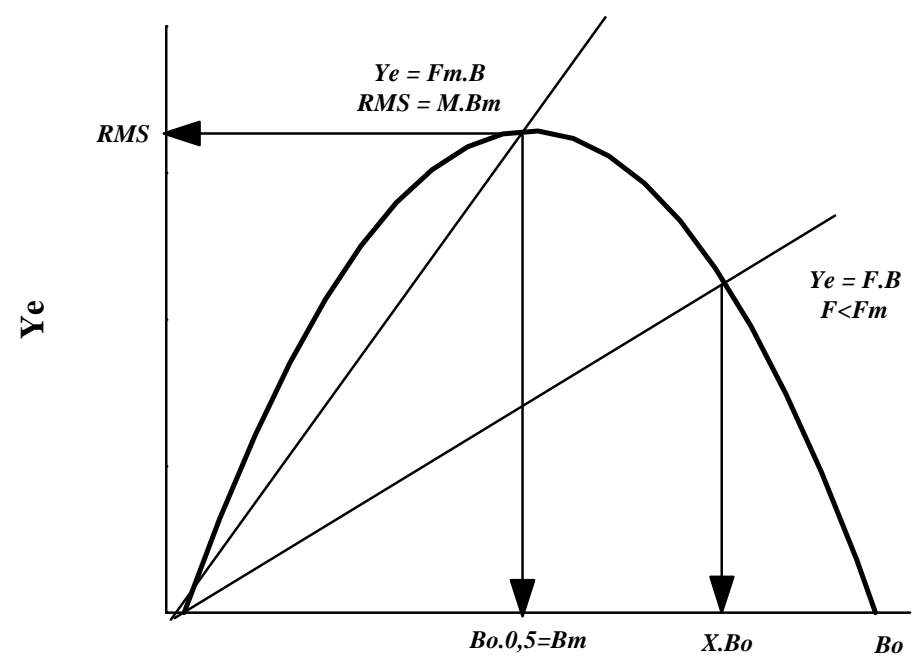

Figura 1. Representação esquemática da relação entre o rendimento em equilíbrio de uma pescaria $Y_{e}$ e a biomassa do estoque $B$, segundo o Modelo de Dinâmica da Biomassa de Graham-Schaefer. O modelo propõe que o Rendimento Máximo Sustentável $(R M S)$ de uma pescaria é obtido quando a biomassa do estoque $\left(B_{m}\right)$ é aproximadamente a metade da biomassa do estoque virginal $B_{0}$. (fator de correção $X=0,5$ ). De acordo com os pressupostos clássicos do modelo de crescimento populacional assumido (logístico) a taxa de mortalidade por pesca que produzirá RMS $\left(F_{m}\right)$ é igual à mortalidade natural $M$.

captura máxima recomendada de $2.500 \mathrm{t} \mathrm{em}$ 2002 ou cerca de $4 \%$ da biomassa original. A escolha de $X=0,27$ teve como justificativa o cenário mencionado acima que prioriza a sustentabilidade das áreas de maior concentração do recurso e efetivamente explotadas em 2001. Especificamente, o valor deriva da razão entre a taxa de remoção total (16\%) e aquela observada dentro das áreas de explotação (60\%). A captura máxima sugerida para 2002 implica em uma redução de 70\% dos níveis de captura de 2001 e que levaria em 2002 a uma taxa de remoção total, dentro das áreas explotadas de $16 \%$.

O estado e as tendências da pescaria de $L$. budegassa no Atlântico NE em decorrência das estratégias de manejo implementadas entre 1983 e 1993 oferecem alguns parâmetros de comparação em suporte aos níveis referenciais de captura de $L$. gastrophysus propostos acima (ICES, 1995). A captura média dessa espécie oscila em tor- no das $8.000 t$ anuais representando cerca de $21 \%$ da biomassa desovante $(41.000 \mathrm{t})$. Considerando que o referido estoque vem apresentado um contínuo declínio e que essa taxa de remoção é induzida através de uma quota anual, fica evidente que a mesma tem sido sobredimensionada para as capacidades de sustentação do estoque. Em 2001 as capturas de L. gastrophysus atingiram cerca de $28 \%$ da biomassa desovante total estimada (31.600 t) e passariam, a partir da captura máxima recomendada acima para cerca de $8 \%$. Enquanto a medida pareceria extremamente conservativa, em se considerando o prioritário cenário de sustentação dentro das áreas de pesca, onde se concentram apenas partes da população (cerca de $1 / 4$ ), na verdade teríamos uma taxa de remoção próxima de $30 \%$, ou seja, ainda elevada em se considerando as tendências do estoque europeu (ICES, 1995).

As taxas de remoção da população desovante recomendadas parecem manter co- 
A

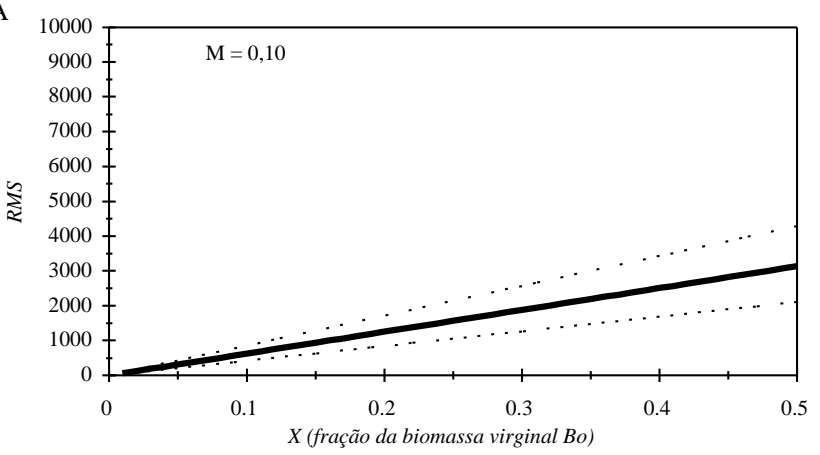

- mediana · . IC 98\%

B

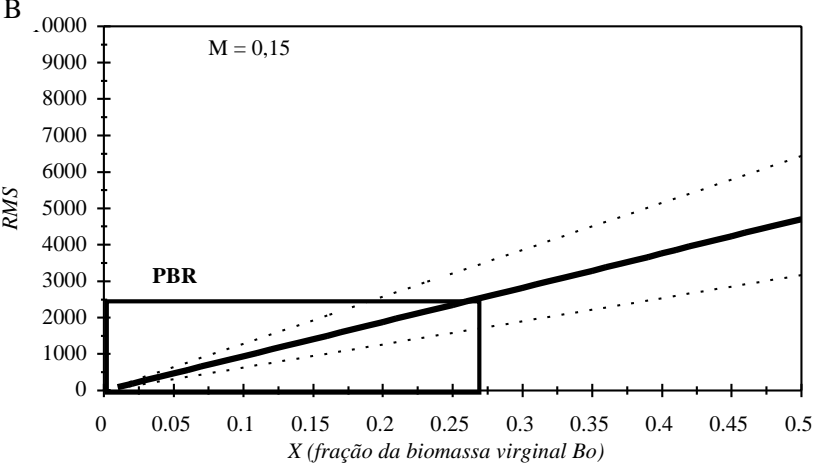

- mediana * . IC 98\%

C

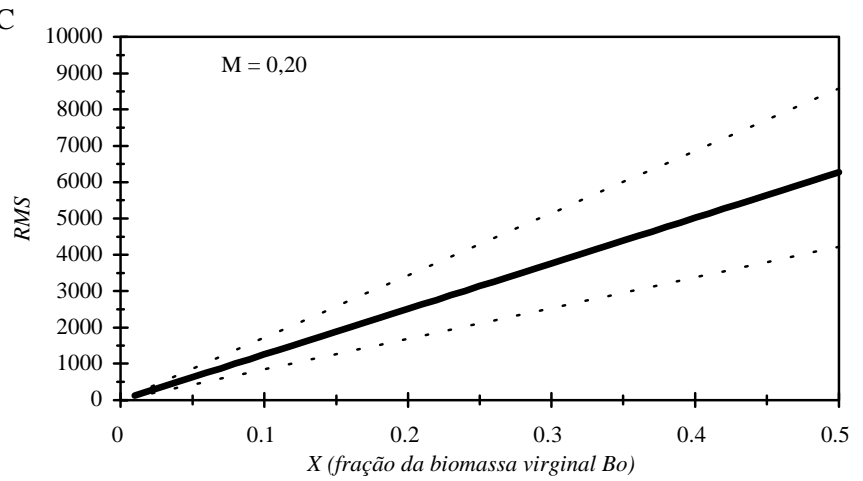

- mediana - - IC $98 \%$

Figura 2. Variação da estimativa do Rendimento Máximo Sustentável (RMS) do peixe-sapo (Lophius gastrophysus) no Sudeste e Sul do Brasil a partir do modelo de Graham-Schaefer e a relação RMS = X.M. $B_{0}$, onde se considerou a mortalidade natural $\mathrm{M}$ variando entre $0,1(\mathrm{~A}), 0,15(\mathrm{~B})$ e $0,2(\mathrm{C})$. $\mathrm{B}_{\mathrm{o}}$ corresponde à biomassa virginal. 
erência com o tempo médio de reposição da parcela removida da população devido à atividade pesqueira. Este tempo tem sido utilizado como um PBR para alguns estoques bentônicos (Orensanz et al., 2000). Sob essa ótica teríamos que fêmeas de peixe-sapo demorariam, segundo parâmetros de crescimento estimados para L. budegassa (Duarte et al., 2001), cerca de 9 anos para atingir a primeira maturação e 11 anos para atingir o tamanho de $60 \mathrm{~cm}$, que correspondeu a parcela dominante em biomassa do estoque explotado no Sudeste e Sul do Brasil (Perez et al., 2002b). Assim, considerando esses ritmos de crescimento, pode-se inferir que a parcela populacional removida pela pesca demoraria em média 9 anos (a uma taxa de $11 \%$ ao ano) e 11 anos (a uma taxa de $9 \%$ ao ano) para ser completamente reposta até a primeira maturação e até a idade em que biomassa disponível à pesca é mais abundante respectivamente. Essas taxas implicariam em capturas anuais máximas de 3.500 te 2.800 t respectivamente, ou seja, próximas das recomendadas neste estudo (Fig. $3)$.

Restrições de natureza biológico-populacionais

Normalmente quando são adotados PBRs baseados em modelos de dinâmica de biomassa são desconsiderados, pela própria natureza dos modelos, elementos populacionais importantes como a estrutura de tamanhos, a proporção de sexos e os padrões de maturação. Associados ao pressuposto do recrutamento constante, isso implica dizer que alterações na estrutura populacional e no recrutamento, especialmente decorrentes da própria atividade de pesca, tendem a alterar a capacidade de reposição de biomassa de uma população, invalidando os PRBs adotados e induzindo a adoção de estratégias inapropriadas de manejo. Um exemplo disso é a conhecida alteração do equilíbrio observada na pescaria de arrasto do camarão-rosa (Farfantepenaeus spp.) no Sudeste do Brasil, em decorrência de pescarias artesanais direcionadas a subadultos no interior de lagunas costeiras e estuários. Essas pescarias, estabelecidas na década de 1980, claramente afetaram o recrutamento do estoque às áreas reprodutivas localizadas na plataforma continental, invalidando, por vários anos, os RMS adotados historicamente para o controle do esforço pesqueiro (Valentini et al., 1991).

No caso do peixe-sapo, a eficiência do PBR adotado tem uma evidente relação com a expectativa de um recrutamento constante. Em 2001 pouco mais da metade da remoção total do estoque de peixe-sapo correspondeu a indivíduos imaturos com destaque para as fêmeas, cuja proporção de imaturos na captura da pesca de arrasto atingiu cerca de $80 \%$ (Schwingel \& Andrade, 2002). Apesar de não se dispor de estimativas de crescimento para L. gastrophysus, existem fortes evidências, a julgar pela biologia das espécies similares do Atlântico, que as fêmeas devem levar de seis a oito anos para atingir o tamanho de primeira maturação (Duarte et al., 1997; Duarte et al., 2001; Landa et al., 2001). Desta forma inferese que a pescaria em 2001 tendeu a afetar o recrutamento futuro do estoque que possivelmente terá, mantidas as práticas de 2001, um potencial de reposição da biomassa removida pela pesca inferior ao previsto neste estudo, além de fundamentalmente invalidar o PBR aqui considerado (Clark, 2001). Assim, é essencial que na captura máxima proposta de $2.500 \mathrm{t}$ a proporção de indivíduos juvenis seja mínima, deslocando a mortalidade por pesca para parcelas pós-reprodutivas do estoque $(>50 \mathrm{~cm}$ de comprimento total).

Sob o aspecto de seletividade, a pesca de emalhe, nos atuais moldes operacionais, apresenta-se compatível com os padrões observados de maturação. Por outro lado, a pesca de arrasto duplo parece ter poucas possibilidades tecnológicas para evitar a captura de peixes-sapo juvenis (Wahrlich \& Rodrigues-Ribeiro, 2002). Em termos ideais seria recomendável que a pesca do peixe-sapo fosse em boa 


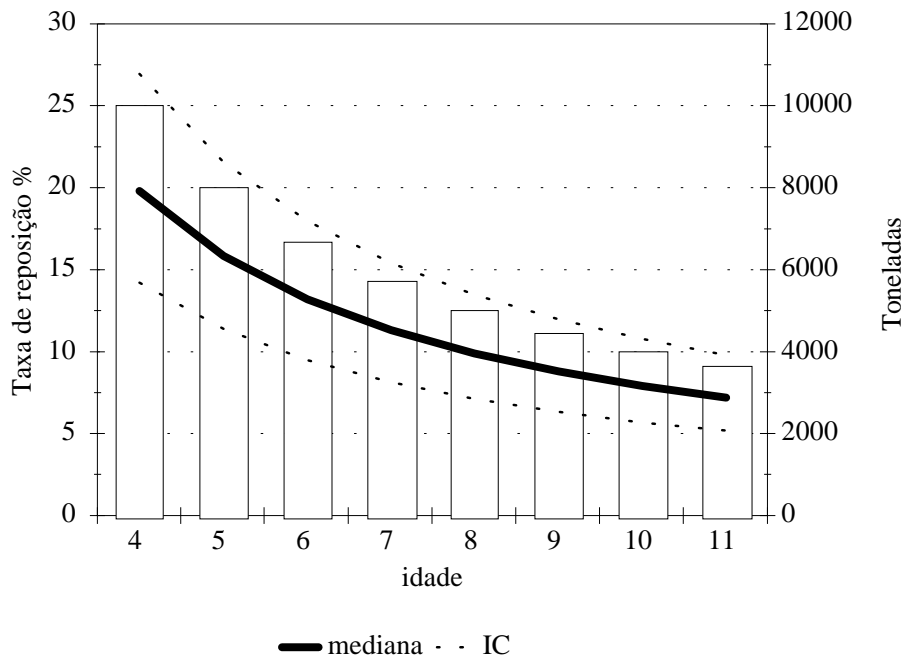

Figura 3. Taxas médias percentuais e absolutas estimadas para reposição do estoque em função da idade e dos ritmos de crescimento calculados para Lophius budegassa no Atlântico NE. Note que se considerando a aparente semelhança entre essa espécie e $L$. gastrophysus o tempo necessário para atingir a primeira maturação e a idade em que existe a maior biomassa disponível para a pesca seriam 9 e 11 anos respectivamente.

parte restrita ao método de rede de emalhe de fundo, o que poderia envolver tanto a manutenção de um número negociável das embarcações atuantes no Sudeste e Sul, seja por arrendamento ou nacionalização das mesmas, ou ainda por um processo de adaptação de parte dos arrasteiros nacionais, na tentativa de se estruturar uma frota nacional de pesca de emalhe dirigida ao peixe-sapo. Caso esse processo seja inviável em médio prazo, é certo que a sobrevivência da frota de arrasto duplo na pescaria futura do peixe-sapo apenas poderá ser tolerada, do ponto de vista do equilíbrio do estoque, se as operações de pesca forem realizadas em estratos de profundidade maiores de 250 m onde seriam menores as chances de captura de indivíduos juvenis. Essa restrição implicaria ainda em licenciamento específico para a espécie e o rastreamento via-satélite das embarcações, nos moldes do que já ocorre com a frota arrendada (Wahrlich \& Rodrigues-Ribeiro, 2002).
Restrições à captura de fauna acompanhante

O impacto da captura de fauna acompanhante na pesca de emalhe direcionada ao peixe-sapo sobre populações com e sem interesse comercial no Sudeste e Sul do Brasil durante 2001 parece ter sido significativo. Particularmente preocupante foi a mortalidade de espécies grandes e suscetíveis a sobrepesca e/ou reduções populacionais importantes, como o cherne-poveiro e elasmobrânquios. Devido aos recentes diagnósticos sobre a situação populacional do cherne-poveiro (Peres \& Haimovici, 1998), torna-se urgente a implementação de medidas que reduzam a captura e o rejeito da espécie na pesca de emalhe, de forma análoga às anteriormente adotadas para a frota arrendada de arrasto (DPA/MAPA, 200; Perez et al. 2001a).

Outro aspecto importante se refere à tendência do direcionamento da pesca de emalhe ao caranguejo-de-profundidade. Essa 
espécie já é objeto de uma pescaria direcionada com covos em desenvolvimento no Sudeste e Sul do Brasil (Perez et al. 2001a) a qual demandará, em curto prazo, a definição de políticas pesqueiras específicas, tendo em conta o elevado valor do recurso. Essas políticas deverão se centralizar em definições claras por parte do MAPA sobre "como" será priorizada a exploração de caranguejo no futuro. Embora a biologia populacional e a pescaria desse recurso estejam em processo de avaliação por parte do Grupo de Estudos Pesqueiros, parece ser recomendável priorizar a captura altamente seletiva obtida com o emprego de covos, em detrimento de um padrão exploratório misto determinado pelas significativas capturas resultantes das redes de emalhe, atualmente direcionadas ao peixe-sapo, em conjunto com as capturas de covos.

A produção de fauna acompanhante pela pesca de emalhe, em função da elevada vulnerabilidade de várias espécies demersais de interesse comercial, tem sido globalmente avaliada no sentido de compatibilizar essa atividade com outras importantes pescarias (Alverson et al., 1994). Na costa leste do Canadá, $L$. americanus tem sido alvo de uma pequena pescaria de emalhe a qual foi submetida, durante sua fase de desenvolvimento, ao manejo através de um regime de quotas anuais de captura. Apesar da captura anual da espécie ser modesta, nunca atingindo as quotas estabelecidas, constatou-se a elevada captura incidental de espécies intensamente explotadas na região como o bacalhau, o haddock e os linguados. Desta forma o regime de quotas foi totalmente abandonado e atualmente a pescaria é manejada unicamente através de critérios de limitação de fauna acompanhante (Kulka \& Miri, 2001). Parece evidente que esses critérios devem fazer parte também do manejo da pescaria de L. gastrophysus no Sudeste e Sul do Brasil.

$\mathrm{Na}$ aparente inviabilidade de soluções tecnológicas para a minimização da captura incidental, deveria ser considerada a possibilidade de fechamento permanente ou temporá- rio das áreas de pesca localizadas entre $28^{\circ} \mathrm{e}$ $30^{\circ} \mathrm{S}$ à pesca de emalhe do peixe-sapo. Essa medida minimizaria o impacto da pesca sobre as concentrações de cherne-poveiro, altamente vulneráveis nessa área entre abril e setembro devido à atividade reprodutiva (Peres, 2000), além de outras espécies altamente suscetíveis e claramente mais vulneráveis na região sul do país com destaque para os cações, arraias, aves, mamíferos marinhos e quelônios (Haimovici et al., 1998; Pinedo, 1998, Peres e Haimovici, 1998). Além disso, a medida poderia também contribuir com a pescaria da própria espécie nas outras regiões já que (a) limitaria a parcela do estoque exposta à taxa de exploração; (b) amenizaria os erros de manejo devido às incertezas nas estimativas de níveis sustentáveis de exploração e nos processos de implementação das medidas de ordenamento; (c) serviria como forma de se monitorar comparativamente os padrões do ciclo de vida de parcelas sujeitas e não sujeitas a explotação pesqueira e (d) potencialmente proporcionaria melhores rendimentos nas demais áreas disponíveis à pesca (Perry et al., 1999). A alternativa é viável do ponto de vista de controle haja vista a eficiência dos sistemas de rastreamento já utilizados para esse fim (Rodrigues-Ribeiro, 2002).

\section{Restrições a práticas de bordo prejudiciais ao} ecossistema marinho

Nas operações de pesca de emalhe direcionadas ao peixe-sapo constataram-se, na maioria das embarcações, práticas de rejeito de sólidos não-biodegradáveis ao oceano incompatíveis com a legislação nacional e internacional. Em particular foram preocupantes os eventuais lançamentos ao mar de fragmentos de panagens de redes danificadas (Wahrlich \& Rodrigues-Ribeiro, 2002). Essas redes, além do evidente efeito poluidor dada a nãodegradabilidade nesse ambiente, podem provocar diversos níveis de pesca fantasma, prejudicial a muitas populações marinhas inclusive ao recurso peixe-sapo. Essa prática é mun- 
dialmente condenada (FAO, 1995) e deveria ser completamente erradicada nas embarcações direcionadas à pesca de peixe-sapo em 2002 seja através da utilização de incineradores e/ ou da estocagem para descarga e destinação adequada em terra.

\section{RECOMENDAÇÕES PARA O ORDENAMENTO DA PESCARIA}

A partir do diagnóstico e dos procedimentos e critérios adotados para a estimativa de níveis sustentáveis de explotação do recurso, foram formuladas nove recomendações objetivas a serem consideradas quando da tomada de decisão sobre o ordenamento da pescaria do peixe-sapo no Sudeste e Sul do Brasil em 2002:

1. Recomenda-se que o desembarque de peixe-sapo no Sudeste e Sul do Brasil em 2002 seja limitado a um máximo de 2.500 toneladas (peixe inteiro eviscerado).

2. Recomenda-se que a captura do peixe-sapo seja realizada apenas por embarcações licenciadas exclusivamente para esse fim, mantendo-se e ampliando-se as exigências de observadores de bordo e sistemas de rastreamento via satélite. Esses sistemas deverão obrigatoriamente permitir o acompanhamento contínuo das posições e profundidades de pesca mediante sua conexão direta com equipamentos receptores de GPS e ecossondas a bordo.

3. Recomenda-se que a pesca do peixe-sapo seja restrita à captura de indivíduos maiores de $50 \mathrm{~cm}$ de comprimento.

4. Recomenda-se que a captura do peixe-sapo seja efetuada em 2002 prioritariamente através de redes de emalhe, a partir da isóbata dos $250 \mathrm{~m}$ e utilizando-se redes com tamanho de malha nunca inferior ao praticado em 2001, ou seja, $280 \mathrm{~mm}$ medidos entre nós opostos com a malha esticada.
5. Recomenda-se que, caso seja mantida a pesca de peixe-sapo através do arrasto de fundo, a mesma seja limitada a profundidades maiores que $250 \mathrm{~m}$ e com redes com tamanho de malha no ensacador nunca inferior a $90 \mathrm{~mm}$ medidos entre nós opostos com malha esticada, visando à redução da captura de indivíduos juvenis. Da mesma forma, recomenda-se que sejam mantidos os limites máximos de captura do cherne-poveiro (Polyprion americanus) e batata (Lopholatilus villari) atualmente em vigor para a frota arrendada.

6. Recomenda-se que o sistema de rotação de áreas de pesca de emalhe adotado em 2001 seja mantido em 2002 e aplicado a todas as embarcações de pesca de emalhe engajadas na pesca do peixe-sapo.

7. Recomenda-se que a área localizada entre $28^{\circ}$ e $30^{\circ} \mathrm{S}$ e limitada em seu bordo leste pela isóbata de $100 \mathrm{~m}$ seja fechada permanentemente ou pelo menos entre os meses de abril e setembro, com vistas à redução da captura incidental do cherne-poveiro (Polyprion americanus) e outras espécies comercialmente importantes e/ou altamente suscetíveis à pesca de emalhe. Se adotado o fechamento temporário da referida área, recomenda-se que a mesma seja automaticamente incluída no sistema de rotação de áreas durante os períodos em que a captura for permitida.

8. Considerando-se a legislação nacional e internacional e os evidentes efeitos negativos ocasionados pela liberação de resíduos de bordo e artefatos de pesca danificados ou em desuso sobre o ecossistema marinho (incluindo a possibilidade de pesca fantasma), recomenda-se que seja implementada, em toda a frota licenciada para a pesca do peixe-sapo no Sudeste-Sul do Brasil, a obrigatoriedade de incineração e/ou armazenamento dos resíduos sólidos não-biodegradáveis a bordo para posterior destinação adequada em terra. 


\section{APRESENTAÇÃO PÚBLICA DOS RESUL- TADOS E RECOMENDAÇÕES}

A etapa seguinte no processo de ordenamento da pescaria do peixe-sapo consistiu da apresentação pública de todo o conhecimento gerado a partir (a) da coleta intensiva de informações tecnológicas, biológicas e pesqueiras, (b) do processamento e análise dessas informações, (c) dos procedimentos e critérios adotados para a estimativa de níveis sustentáveis de explotação do recurso e, (d) do estabelecimento, através de recomendações, de padrões sustentáveis de explotação do peixe-sapo. Essa apresentação foi realizada na UNIVALI no dia 25 de abril de 2002, no formato de um seminário intitulado "Avaliação da Pescaria do Peixe-sapo no Sudeste e Sul do Brasil" o qual foi estruturado em palestras proferidas pelos pesquisadores sobre cada um dos aspectos estudados (ANEXO I) e contou com ampla participação de setores envolvidos com a atividade pesqueira nas regiões Sudeste e Sul do Brasil incluindo quatro instituições de pesquisa pesqueira, oito empresas de pesca, onze sindicatos e duas confederações de pescadores e empresários da pesca, sete instituições públicas atuantes nas áreas ambientais e agropecuárias, três emissoras de televisão, acadêmicos e público em geral (ANEXOII).

O seminário, e o debate aberto por ele gerado, cumpriu uma etapa básica de esclarecimento amplo da situação e das perspectivas de explotação sustentável do recurso peixesapo, atendendo compromissos para com o MAPA e principalmente para com o setor pesqueiro que disponibilizou informações pesqueiras e/ou viabilizou, inclusive com o investimento de recursos financeiros, a possibilidade de coleta detalhada dessas informações (i.e. contratação de observadores de bordo). Além da conscientização do setor quanto à responsabilidade de se preservar um recurso valioso e da própria existência dessa nova alternativa pesqueira, esse processo de esclarecimento auxiliou na preparação do setor para uma pos- terior negociação das medidas de ordenamento dentro do âmbito do CPG de Demersais de Profundidade (DPA-MAPA) onde o setor se faz representado por suas entidades de classe. A transparência do processo incrementou a expectativa de uma negociação democrática, participativa e responsável o qual deverá resultar na tomada de decisões eqüitativas e dentro de uma abordagem de precaução.

\section{AGRADECIMENTOS}

Os participantes da análise da pescaria do peixe-sapo no Sudeste e Sul do Brasil agradecem a inestimável colaboração de todos os observadores de bordo do PROA, das equipes de campo, informática e processamento do Programa de Estatística Pesqueira Industrial de Santa Catarina e SIESPE (Sistema Integrado de Estatística Pesqueira) e da administração do CTTMar-UNIVALI, sem os quais o trabalho não seria possível. Um agradecimento especial vai para o Cmte. Flávio Leme (Sindicato dos Armadores de Pesca do Rio de Janeiro -SAPERJ) e Marcus Carneiro (Instituto de Pesca, IP-Santos ) pelos aportes às estatísticas de desembarque do peixe-sapo em 2001. Da mesma maneira, agradecemos a todos os empresários, mestres, pescadores, funcionários de empresas pesqueiras e Sindicatos que de alguma maneira colaboraram e confiaram no nosso trabalho.

\section{REFERÊNCIAS BIBLIOGRÁFICAS}

Alverson, D.L.; Freeberg, M.G.; Murawsky, S.A. \& J.G. Pope. 1994. A global assessment of fisheries bycatch and discards. FAO Fish. Tech. Pap. 330. 233p.

Bruno, I.; Fariña, A.C.; Landa, J. \& R. Morlán. 2001. The Gillnet fishery for Anglerfish (Lophius piscatorius) in Deep-water in the Northwest of Iberian Peninsula. NAFO SCR Doc. 01/99. Serial No. N4487 
Caddy, J.F. 1999. Deciding on precautionary management measures for stock based on a suite of Limit Reference Points (LRPs) as a basis for a Multi-LRP harvest law. NAFO Sci. Coun. Studies, 32:55-68.

Clark, M. 2001. Are deepwater fisheries sustainable? - the example of orange roughy (Hoplostethus atlanticus) in New Zealand. Fish. Res. 51:123-135.

DPA/MAPA, 2000. Reunião Consultiva para um exame da Situação dos Recursos Pesqueiros Demersais da Região Sudeste-Sul. Disponível em: http://www.gep.cttmar.univali.br Acesso em 23 Abr. 2002.

Duarte, R.; Azevedo, M. \& P. Pereda. 1997. Study of the growth of southern black and White monkfish stocks. ICES J. Mar. Sci., 54:866-874.

Duarte. R.; Azevedo, M.; Landa, J. \& P. Pereda. 2001. Reproduction of anglerfish (Lophius budegassa Spinola ans Lophius piscatorius Linnaeus) from the Atlantic Iberian coast. Fish. Res. 51:349-361.

FAO, 1995. Código de conducta para la pesca responsable. FAO, Roma, 45p.

Garcia, S. 1996. The precautionary approach to fisheries and its implications for fishery research, technology and management: an updated review, p. 1-65. In FAO. Precautionary approach to fisheries. Part 2: Scientific papers. Prepared for the Technical Consultation on the Precautionary Approach to Capture Fisheries (including species introduction). Lysekil, Sweden, 6-13 June 1995. (A scientific meeting organized by the Government of Sweden in cooperation with FAO).

Garcia, S.; Sparre, P. \& J. Csirke. 1989. Estimating surplus production of maximum sustainable yield from biomass data when effort time series are not available. Fish. Res. 8:13-23.

Gulland, J.A. 1971. The fish resources of the ocean. Fishing News (Books) Limited Publishers, West Byfleet, Surrey.
Haimovici, M.; Castello, J.P. \& C.M. Vooren., 1997. Fisheries, p. 183-196. In U. Seeliger, C. Odebrecht \& J.P. Castello (Eds.) Subtropical Convergence Environments. The coast and sea in the Southwestern Atlantic. Springer Verlag, Berlin.

Kulka, D.W. \& C.M. Miri. 2001. The status of monkfish (Lophius americanus) in NAFO Divisions $2 \mathrm{~J}, 3 \mathrm{~K}, 3 \mathrm{~L}, 3 \mathrm{~N}, 3 \mathrm{O}$ and subdivisions 3Ps. NAFO SCR Doc. 01/47. Serial No. N4425, 33p.

Landa, J.; Pereda, P.; Duarte, R. \& M. AZEVEDO. 2001. Growth of anglerfish (Lophius piscatorius and L. budegassa) in Atlantic Iberian waters. Fish. Res. 51:363-376.

Peres, M. B. 2000. A biologia do cherne-poveiro (Polyprion americanus). In. DPA/MAPA. Reunião Consultiva para um exame da Situação dos Recursos Pesqueiros Demersais da Região Sudeste-Sul. Disponível em: http://www.gep.cttmar.univali.br . Acesso em 23 Abr. 2002.

Peres, M.B. \& M. Haimovici. 1998. A pesca dirigida ao cherne-poveiro Polyprion americanus (Polyprionidae, Teleostei) no sul do Brasil. Atlântica, Rio Grande 20:141161.

Perez, J. A. A.; Wahrlich, R.; Pezzuto, P. R.; Schwingel, P.R.; Lopes, F.R.A.\& M. Rodrigues-Ribeiro. 2001a. Deep-sea fishery off southern Brazil: Recent trends of the Brazilian fishing industry. NAFO SCR Doc. 01/117. Serial No. N4505.21p.

Perez, J.A.A.; Pezzuto, P.R.; Rodriguez, L.F.; Valentini, H \& C.M. Vooren. 2001b. Relatório da reunião técnica de ordenamento da pesca demersal nas regiões Sudeste e Sul do Brasil. In P.R. Pezzuto; J.A.A. Perez; L.F. Rodrigues; H. Valentini (Eds.), Reuniões de Ordenamento da Pesca Demersal no Sudeste e Sul do Brasil: 2000-2001. Notas Téc. FACIMAR, 5:1-34.

Perez, J.A.A.; Andrade, H.A.; Pezzuto, P.R.; Rodrigues-Ribeiro, M.; Schwingel, P.R. \& R. Wahrlich. 2002a. Análise da Pescaria do Peixe-sapo no Sudeste e Sul do Brasil 
- Ano 2001. Convênio Ministério da Agricultura, Pecuária e Abastecimento - Universidade do Vale do Itajaí (MAPA/SARC/ DPA 03-2001). Relatório Final, Itajaí-SC, Abril de 2002.

Perez, J.A.A.; Wahrlich, R.; Pezzuto, P.R.; M. Rodrigues-Ribeiro. 2002b. Capítulo 2: Estrutura e dinâmica da pescaria do peixesapo. In J.A.A. Perez; H.A. Andrade; P.R. Pezzuto; M. Rodrigues-Ribeiro; P.R. Schwingel \& R. Wahrlich. Análise da Pescaria do Peixe-sapo no Sudeste e Sul do Brasil-Ano 2001. Convênio Ministério da Agricultura, Pecuária e Abastecimento Universidade do Vale do Itajaí (MAPA/ SARC/DPA 03-2001). Relatório Final, ItajaíSC, Abril de 2002.

Perez, J.A.A.; Pezzuto, P.R. \& H.A. Andrade. 2002c. Capítulo 4: Avaliação de Estoque. In J.A.A. Perez; H.A. Andrade; P.R. Pezzuto; M. Rodrigues-Ribeiro; P.R. Schwingel \& R. Wahrlich. Análise da Pescaria do Peixe-sapo no Sudeste e Sul do Brasil - Ano 2001. Convênio Ministério da Agricultura, Pecuária e Abastecimento Universidade do Vale do Itajaí (MAPA/ SARC/DPA 03-2001). Relatório Final, ItajaíSC, Abril de 2002.

Perry, R.I.; Walters, C.J. \& J.A. Boutilier. 1999. A framework for providing scientific advice for the management of new and developing invertebrate fisheries. Rev. Fish Biol. Fish., 9:125-150.

Pinedo, M.C. 1998. Mamíferos e tartarugas marinhas, p. 166-170. In U. Seeliger, C. Odebrecht \& J.P. Castello (Eds.), Subtropical Convergence Environments. The coast and sea in the Southwestern Atlantic. Springer Verlag, Berlin.

Quinn II, T.J. \& R.B. Deriso. 1999. Quantitative Fish Dynamics. Oxford University Press. Oxford, 542p.

Rodrigues-Ribeiro, M., 2002. Manutenção de uma Central de Controle de Rastreamento por Satélite de Embarcações Arrendadas. Meta 04, Relatório Final Ações Prioritárias ao Desenvolvimento da Pesca e Aqüicultura no Sul do Brasil, Convênio Ministério da Agricultura, Pecuária e Abastecimento (MAPA), Universidade do Vale do Itajaí, MAPA/SARC/DPA/ No.003/2001.

Schwingel, P.R. \& H.A. Andrade. 2002. Capítulo 3: Aspectos biológicos e populacionais. In J.A.A. Perez; H.A. Andrade; P.R. Pezzuto; M. Rodrigues-Ribeiro; P.R. Schwingel \& R. Wahrlich. Análise da Pescaria do Peixe-sapo no Sudeste e Sul do Brasil - Ano 2001. Convênio Ministério da Agricultura, Pecuária e Abastecimento - Universidade do Vale do Itajaí (MAPA/SARC/DPA 03-2001). Relatório Final, Itajaí-SC, Abril de 2002.

Wahrlich, R. 2002. Programa de Observadores de Bordo em Embarcações Arrendadas. Meta 03, Relatório final Ações Prioritárias ao Desenvolvimento da Pesca e Aquicultura no Sul do Brasil, Convênio Ministério da Agricultura, Pecuária e Abastecimento (MAPA), Universidade do Vale do Itajaí, MAPA/SARC/DPA No 003/2001.

Wahrlich, R. \& M. Rodrigues-Ribeiro. 2002. Capítulo 1: Tecnologia da Pesca. In J.A.A. Perez; H.A. Andrade; P.R. Pezzuto; M. Rodrigues-Ribeiro; P.R. Schwingel \& R. Wahrlich. Análise da Pescaria do Peixesapo no Sudeste e Sul do Brasil - Ano 2001. Convênio Ministério da Agricultura, Pecuária e Abastecimento - Universidade do Vale do Itajaí (MAPA/SARC/DPA 032001). Relatório Final, Itajaí-SC, Abril de 2002.

Woodby, D.A.; Gorden, H.K. \& R.C. Larson. 1993. A conservative application of a surplus production model to the seacucumber fishery in southeast Alaska, $p$. 191-201. In G. Kruse; D.M. Eggers; R.J. Marasco; C. Pautzke \& T.J. Quinn II (Eds.) Proceedings of the International Symposium on Management Strategies for Exploited Fish Populations. Lowell Wakefield Fisheries Symposium series. Alaska Sea Grant College Program Report, No. 93-2. 
Valentini, H.; D'Incao, F.; Rodrigues, L. F.; Rebelo Neto, J. E. \& E. Rahn. 1991. Análise da pesca do camarão-rosa (Penaeus brasiliensis e Penaeus paulensis) nas regiões sudeste e sul do Brasil. Atlântica, Rio Grande 13(1): 143-158. 
ANÁLISE DA PESCARIA DO PEIXE-SAPO Lophius gastrophysus NO SUDESTE E SUL DO BRASIL - ANO 2001 Grupo de Estudos Pesqueiros (CTTMar - UNIVALI)

Departamento de Pesca e Aqüicultura (DPA)

Ministério da Agricultura, Pecuária e Abastecimento (MAPA)

Convênio MAPA/SARC/DPA 03-2001

UNIVALI, ITAJAÍ - SC - 25 de abril de 2002

\begin{tabular}{|c|c|c|}
\hline \multicolumn{3}{|c|}{ AGENDA } \\
\hline Horário & & \\
\hline $08: 30 \mathrm{~h}-09: 00 \mathrm{~h}$ & Abertura & $\begin{array}{c}\text { DPA/MAPA } \\
\text { CTTMar/UNIVALI } \\
\text { Setor Produtivo SE-S }\end{array}$ \\
\hline 09:00 h-10:00 h & $\begin{array}{l}\text { O processo de ordenamento da pescaria do } \\
\text { peixe-sapo no Sudeste e Sul do Brasil }\end{array}$ & $\begin{array}{c}\text { Gabriel Calzavara } \\
\text { Diretor } \\
\text { DPA - MAPA } \\
\end{array}$ \\
\hline $10: 00 \mathrm{~h}-10: 30 \mathrm{~h}$ & Café & \\
\hline $10: 30 \mathrm{~h}-11: 00 \mathrm{~h}$ & $\begin{array}{l}\text { A análise da pescaria do peixe-sapo em 2001: } \\
\text { fontes de informação, dados obtidos, métodos e } \\
\text { critérios }\end{array}$ & $\begin{array}{l}\text { Dr. José Angel Alvarez Perez } \\
\text { Coordenador da Análise da pescaria do peixe-sapo em } \\
\qquad 2001 \\
\text { Convênio UNIVALI/MAPA } \\
\text { CTTMar - UNIVALI }\end{array}$ \\
\hline $11: 00 \mathrm{~h}-12: 00 \mathrm{~h}$ & $\begin{array}{l}\text { A tecnologia empregada na pesca do peixe-sapo: } \\
\text { métodos e operações de pesca, processamento, } \\
\text { rastreamento e práticas à bordo }\end{array}$ & $\begin{array}{c}\text { MSc. Roberto Wahrlich } \\
\text { Pesquisador-Colaborador da Análise da pescaria do peixe- } \\
\text { sapo em } 2001 \\
\text { Convênio UNIVALI/DPA } \\
\text { CTTMar - UNIVALI }\end{array}$ \\
\hline $12: 00 \mathrm{~h}$ & Almoço & \\
\hline $14: 00 \mathrm{~h}-14: 30 \mathrm{~h}$ & $\begin{array}{c}\text { A estrutura e a dinâmica da pesca do peixe-sapo: } \\
\text { ocupação das áreas de pesca, captura, esforço e } \\
\text { fauna acompanhante }\end{array}$ & \begin{tabular}{l}
\multicolumn{1}{c}{ Dr. José Angel Alvarez Perez } \\
Coordenador da Análise da pescaria do peixe-sapo em \\
2001 \\
UNIVALI/MAPA \\
$\qquad$ CTTMar - UNIVALI \\
\end{tabular} \\
\hline $14: 30 \mathrm{~h}-15: 00 \mathrm{~h}$ & $\begin{array}{l}\text { A biologia do peixe-sapo: maturação sexual, } \\
\text { ciclo reprodutivo, estrutura da população e } \\
\text { seletividade das artes de pesca }\end{array}$ & $\begin{array}{l}\text { Dr. Paulo Ricardo Schwingel } \\
\text { Pesquisador-Colaborador da Análise da pescaria do peixe- } \\
\text { sapo em } 2001 \\
\text { Convênio UNIVALI/MAPA } \\
\text { CTTMar - UNIVALI }\end{array}$ \\
\hline $15: 00 \mathrm{~h}-15: 30 \mathrm{~h}$ & Café & \\
\hline $15: 30 h-16: 00 h$ & $\begin{array}{l}\text { Avaliação do tamanho do estoque do peixe-sapo } \\
\text { e da taxa de explotação em } 2001\end{array}$ & $\begin{array}{lc} & \text { Dr. Paulo Ricardo Pezzuto } \\
\text { Pesquisador-Colaborador da Análise da pescaria do peixe- } \\
\text { sapo em } 2001 \\
\\
\text { Convênio UNIVALI/DPA } \\
\text { CTTMar - UNIVALI } \\
\end{array}$ \\
\hline $16: 00 \mathrm{~h}-16: 30 \mathrm{~h}$ & $\begin{array}{l}\text { Diagnóstico do estado da pescaria do peixe-sapo } \\
\text { e recomendações para o ordenamento }\end{array}$ & \begin{tabular}{l}
\multicolumn{1}{c}{ Dr. José Angel Alvarez Perez } \\
Coordenador da Análise da pescaria do peixe-sapo em \\
2001 \\
Convênio UNIVALI/MAPA \\
$\qquad$ CTTMar - UNIVALI
\end{tabular} \\
\hline $16: 30-18: 00 \mathrm{~h}$ & $\begin{array}{c}\text { Debate Aberto } \\
\text { Caminhos para a explotação sustentável do } \\
\text { peixe-sapo }\end{array}$ & $\begin{array}{l}\text { CTTMar/UNIVALI } \\
\text { DPA/MAPA } \\
\text { Setor Produtivo do SE-S } \\
\text { Comunidade em geral }\end{array}$ \\
\hline $18: 00 \mathrm{~h}$ & Encerramento & \\
\hline
\end{tabular}


ANÁLISE DA PESCARIA DO PEIXE-SAPO Lophius gastrophysus NO SUDESTE E SUL DO BRASIL - ANO 2001 Grupo de Estudos Pesqueiros (CTTMar - UNIVALI)

Departamento de Pesca e Aqüicultura (DPA)

Ministério da Agricultura, Pecuária e Abastecimento (MAPA)

Convênio MAPA/SARC/DPA 03-2001

UNIVALI, ITAJAÍ - SC - 25 de abril de 2002

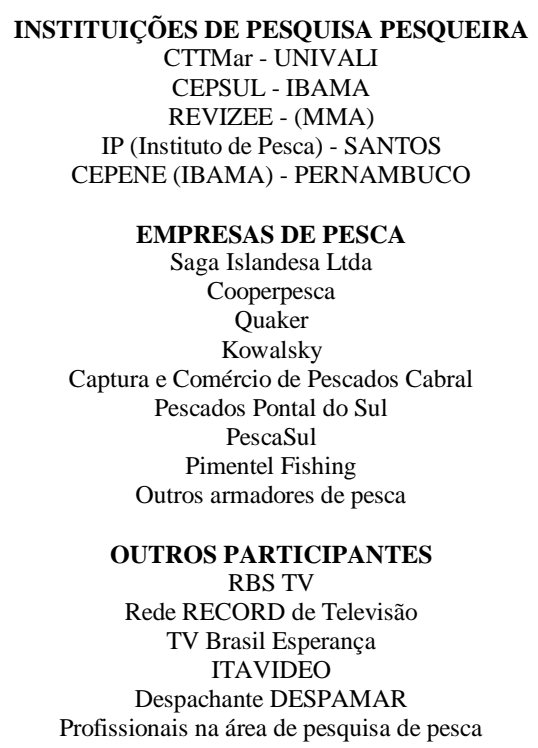

Lista de participantes
Sindicatos dos Pescadores de São Paulo Sindicatos dos Pescadores do Rio de Janeiro Sindicatos dos Pescadores do Espírito Santo Sindicatos dos Pescadores do Rio Grande do Sul Sindicatos dos Pescadores de Santa Catarina

$$
\begin{aligned}
& \text { SITRAPESCA -SC } \\
& \text { SINDIPESCA - RS }
\end{aligned}
$$$$
\text { SINDIPI - SC }
$$

CNP - Confederação Nacional dos Pescadores Sindicato da Indústria da Pesca de Florianópolis SAPERJ - Sind. dos Armadores da Pesca do Rio de Janeiro Sindicato dos Armadores da Pesca de São Paulo CONEPE

\section{OUTRAS INSTITUIÇÕES \\ IBAMA - RJ} IBAMA - DF

SINCOM - Prefeitura Municipal de Itajaí DPA / MAPA

$$
\begin{aligned}
& \text { DFA -MAPA - SC } \\
& \text { DFA - MAPA - PR } \\
& \text { EMATER - PR }
\end{aligned}
$$

